\title{
DESIGN AND TRANSFER OF OsSWEET14-EDITING T-DNA CONSTRUCT TO BAC THOM 7 RICE CULTIVAR
}

\author{
Vu Hoai Sam ${ }^{1}$, Pham Thi $\operatorname{Van}^{2}$, Nguyen Thanh $\mathrm{Ha}^{2}$, Nguyen Thi Thu $\mathrm{Ha}^{2}$, \\ Phung Thi Thu Huong', Pham Xuan $\mathrm{Hoi}^{2}$, Nguyen Duy Phuong,"*, Cao Le Quyen ${ }^{2}$ \\ ${ }^{1}$ National Institute of Medicinal Materials, Ha Noi \\ ${ }^{2}$ Agricultural Genetics Institute, , Ha Noi
}

Received 23 March 2020, accepted 2 February 2021

\begin{abstract}
Bac thom 7 rice (BT7 rice) is one of the major elite rice cultivars in Vietnam with superior productivity and quality but very susceptible to bacterial leaf blight caused by Xanthomonas oryzae pv. oryzae. The gene OsSWEET14, belonging to the OsSWEET family which encodes sugar transport proteins, is considered to be a susceptible gene involved in the virulence of Xoo. At least three cis-elements (EBE - Effector-binding element), including Tal5, PthXo3 and AvrXa7 on BT7 OsSWEET14 promoter, bind specifically to well-known transcription activatorlike effectors (TALEs) of many Asian Xoo bacterium strains. In this study, a T-DNA construct which expressed the protein-RNA complex CRISPR/Cas for editing three EBEs position on the OsSWEET14 promoter was designed. The recombinant binary vector was tested by PCR, restriction enzyme and finally sequencing and then successfully transferred to Bac thom 7 rice through Agrobacterium tumefaciens. 28 of 30 hygromycin-resistant regenerated rice lines that grew and developed normally under nethouse conditions were selected by PCR with specific primers. Among these, twelve transgenic rice lines were identified carrying one copy of the TDNA construct. The presence of CRISPR/Cas9-induced mutations of the targeted promoter in the transgenic plants were confirmed by T7EI assay. These results provide the basis to determine the role of OsSWEET14 in the susceptibility of Bac thom 7 rice to Xanthomonas oryzae pv. oryzae caused disease, towards the further goal of generating an improved Bac thom 7 rice variety with broad-spectrum bacterial leaf blight resistance using CRISPR/Cas 9 technology.
\end{abstract}

Keywords: Bacterial leaf blight, Bac thom 7, CRISPR/Cas9, OsSWEET14, Xanthomonas oryzae.

Citation: Vu Hoai Sam, Pham Thi Van, Nguyen Thanh Ha, Nguyen Thi Thu Ha, Phung Thi Thu Huong, Pham Xuan Hoi, Nguyen Duy Phuong, Cao Le Quyen, 2021. Design and transfer of OsSWEET14-editing T-DNA construct to Bac thom 7 rice cultivar. Academia Journal of Biology, 43(1): 99-108. https://doi.org/10.15625/2615-9023/14923

*Corresponding author email: phuongnd.bio@gmail.com

(O2021 Vietnam Academy of Science and Technology (VAST) 


\section{INTRODUCTION}

Bac thom 7 rice (BT7 rice) is an inbred rice variety originating from China, and recognized according to Decision No. 1224 QD/BNN-KHCN dated April 21, 1998. With some valuable traits such as high yields and good quality, BT7 had been ranked first among popular rice varieties for more than 20 years with over 40 crops in Vietnam. However, its productivity is seriously affected by the bacterial leaf blight disease (BLB), especially in the seasons with heavy rains and strong winds.

BLB disease is caused by the Xanthomonas oryzae pv. oryzae (Xoo) (Borkar, Yumlemban, 2016). The OSSWEET14 gene, a member of the OSSWEET family which encodes sugar transport proteins, is considered a important susceptible gene for Xoo bacteria in rice plants (Streubel et al., 2013). The role of OsSWEET14 in the virulence of several representative Xoo strains collected in the Northern provinces of Vietnam had also been determined through artificial infection experiments (Sam et al., 2019). At the molecular level, Xoo bacteria secrete TAL proteins which activate the targeted gene through the specific binding to cis elements, named as effector-binding element (EBE), located on the promoter region. Some studies on producing rice varieties resistant to Xoo through editing the promoter OsSWEET14 have been published (Li et al., 2012; Blanvillain-Baufumé et al., 2017). These results show that the application of gene editing tools, including the CRISPR/Cas9 system to creating a BLB-resistant BT7 rice variety via editing the susceptible OSSWEETI4 gene is a promising research direction.

In the previous study, we already isolated, sequenced and identified 4 EBEs located on the OSSWEETI4 promoter region of the rice variety BT7 (referred to as SW14BT7), including TalC, AvrXa7, PthXo3 and Tal5 (Sam et al., 2019). However, several studies have demonstrated that EBE TalC is more related to the virulence of African Xoo strains than Asian ones (Blanvillain-Baufumé et al., 2017). Therefore, in this study, to determine the role of the three EBEs AvrXa7, PthXo3 and Tal5 in the infection process of Vietnamese Xoo strains on BT7, a binary vector that carries the construct expressing the modified protein-RNA complexes (SW14-BT7) has been designed and transferred to BT7 rice through $A$. tumefaciens. This study will be the prerequisite for the generation of an improved broad-spectrum BLB-resistant BT7 rice variety by CRISPR/Cas 9 technology.

\section{MATERIALS AND METHODS}

BT7 rice were supplied by Thai Binh Seed Corparation.

Escherichia coli strain DH5 $\alpha$ and Agrobacterium tumefaciens strain EHA105 were purchased from Thermo Fisher Scientific (USA) and Clontech Laboratories (USA), respectively. Vectors pENTR4/gRNA and pCas9 based on the pENTR4 vector (Marker Gene Technologies, USA) and pCAMBIA1300 vector (Invitrogen, USA) were provided by Dr. Sebastien Cunnac (Center for Development Studies, Montpellier, France).

The oligonucleotides (Table 1) used in the study were designed based on sequences published on GenBank and ordered from Invitrogen (USA) and Sigma (USA).

\section{Construction of binary vector for SW14- BT7 modification}

The gRNA sequence simultaneously targeting 3 positions of AvrXa7, PthXo3 and Tal5 EBEs on SW14-BT7 was designed using the CRISPR-P v2.0 software (http://crispr.hzau.edu.cn/CRISPR2/). The rice genomic DNA fragments homologous to the gRNAs were identified using CCTop software (https://crispr.cos.uni-heidelberg.de/). The designed $g R N A$ sequence was synthesized by Invitrogen (USA).

Two 24-nucleotide fragments (carrying 20 nucleotides of gRNA and 4 added nucleotides for sticky 5'-end), namely SW14-gRNA-Fw and SW14-gRNA-Rv were annealed $\left(95^{\circ} \mathrm{C}\right.$ for $\left.10 \mathrm{~min} ., 4{ }^{\circ} \mathrm{C}-\infty\right)$ to create a double stranded 
crRNA (SW14-gRNA). The SW14-gRNA DNA fragment was inserted into the $B s a \mathrm{I}$ position between the OsU6 promoter region and tracRNA on the pENTR4/gRNA vector (Fig. 1). The recombinant vector was confirmed by PCR with the pEN-Fw/pEN-Rv (1.4 kb) and U6-Fw/gRNA-SW14-Rv (276 bp) primer pairs. Next, the fragment containing the [U6:
gRNA-SW14] construct (451 bp) was ligased into the pCas9 binary vector using the Gateway Cloning kit (Invitrogen). The recombinant vector was tested by PCR with Ubi-Fw/NOS-Rv (4.3 kb), Cas9-Fw/Cas9-Rv (4.1 kb), U6-Fw/Ter-Rv (451 bp), U6Fw/gRNA-SW14-Rv (276 bp) primers and finally by sequencing.

Table 1. Oligonucleotides used in the study

\begin{tabular}{|c|c|c|c|}
\hline Name & Sequence $\left(5^{\prime}-3^{\prime}\right)$ & $\begin{array}{l}\text { PCR product } \\
\text { size }(\mathrm{bp})\end{array}$ & Gene/vector \\
\hline Actin-Fw & TGATGGTGTCAGCCACACT & \multirow{2}{*}{350} & \multirow{2}{*}{ Actinl } \\
\hline Actin-Rv & TGGTCTTGGCAGTCTCCATT & & \\
\hline HPT-Fw & AAGGAGGTGATCCAGCC & \multirow{2}{*}{400} & \multirow{2}{*}{$H P T$ (pCas9) } \\
\hline HPT-Rv & GAGTTTGATCCTGGCTCAG & & \\
\hline Ubi-Fw & CCCTGCCTTCATACGCTATT & \multirow{2}{*}{4315} & \multirow{2}{*}{ [Ubi:Nos] (pCas9) } \\
\hline NOS-Rv & AGACCGGCAACAGGATTCAA & & \\
\hline Cas9-Fw & CCATGGCCCCAAAGAAGAAG & \multirow{2}{*}{4108} & \multirow{2}{*}{ Cas9 (pCas9) } \\
\hline Cas9-Rv & TCAATCGCCGCCGAGTTG & & \\
\hline Cas9-t-Rv & GCCTCGGCTGTCTCGCCA & & $\operatorname{Cas} 9$ (pCas9) \\
\hline U6-Fw & GGATCCGGATCATGAACCAACG & \multirow{2}{*}{451} & \multirow{2}{*}{ pENTR4/gRNA } \\
\hline Ter-Rv & GAATTCGATATCAAGCTT & & \\
\hline $\mathrm{pEN}-\mathrm{Fw}$ & CTACAAACTCTTCCTGTTAGTTAG & \multirow{2}{*}{1384} & \multirow{2}{*}{ pENTR4/gRNA } \\
\hline $\mathrm{pEN}-\mathrm{Rv}$ & ATGGCTCATAACACCCCTTG & & \\
\hline $\begin{array}{l}\text { gRNA- } \\
\text { SW14-FW }\end{array}$ & $\begin{array}{l}\text { GTGTGGCTTGATGAGCTTAGCAC } \\
\text { C }\end{array}$ & & \multirow{2}{*}{ gRNA-SW14 } \\
\hline $\begin{array}{l}\text { gRNA- } \\
\text { SW14-Rv }\end{array}$ & $\begin{array}{l}\text { AAACGGTGCTAAGCTCATCAAGC } \\
\text { C }\end{array}$ & & \\
\hline SW14-t-Fw & AACAAAAAAAAGCTAGCAGA & \multirow{2}{*}{520} & \multirow{2}{*}{ SW14-BT7 } \\
\hline SW14-t-Rv & CTTCGCCTTTGGTCTCCTAG & & \\
\hline
\end{tabular}

Note: Underlined sequences denote recognition sites of restriction enzymes.

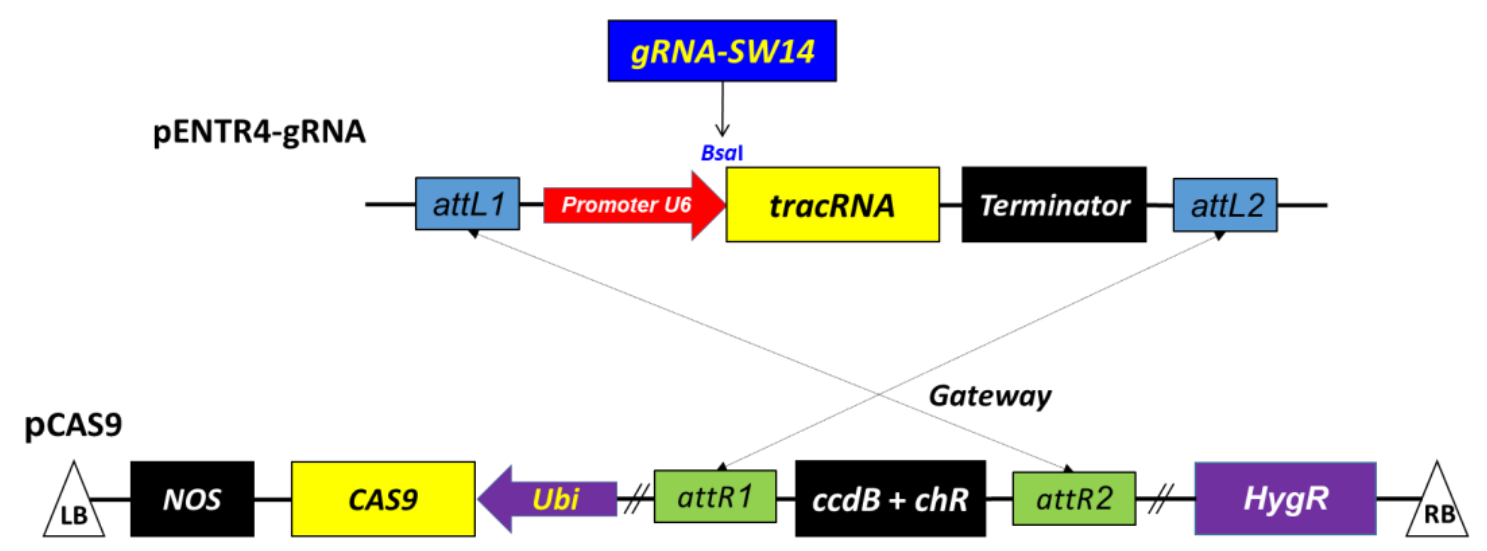

Figure 1. Diagram of constructing pCas9/gRNA-SW14 vector 
Transformation of DNA into A. Tumefaciens

The binary vector was transformed into A. tumefaciens EHA105 by heat shock method (Wang, 2006). Plasmid DNA $(1 \mu \mathrm{g})$ was added to $100 \mu \mathrm{L}$ of competent cells and heat-shocked at $37{ }^{\circ} \mathrm{C}$ for 5 minutes. The reaction mixture was plated on LB medium containing $50 \mu \mathrm{g} / \mathrm{mL}$ spectinomycin, rifamicin $20 \mu \mathrm{g} / \mathrm{mL}$; plates were incubated at $28{ }^{\circ} \mathrm{C}$ for $2-3$ days. The transformants were then screened by colony PCR method (Sahoo et al., 2011) using the U6-Fw/Ter-Rv specific primers.

\section{Rice transformation through A. Tumefaciens}

The rice transformation experiment was performed according to Hiei et al. (1994), with some modifications. Calli formed from BT7 rice endosperm after 5-7 days of culture on MS medium were co-cultured with $A$. tumefaciens bacteria transformed vector pCas9/gRNA-SW14 for three days. Then, the calli were selected on culture media containing $20 \mathrm{mg} / \mathrm{L}$ and $50 \mathrm{mg} / \mathrm{L}$ hygromycin. The surviving calli were transferred in turn to the pre-regeneration medium (MS medium with $5.0 \mathrm{mg} / \mathrm{L} \mathrm{ABA}, 100 \mathrm{mg} / \mathrm{L}$ Cefotaxime and $50 \mathrm{mg} / \mathrm{L}$ Hygromycin), shoot regeneration medium (MS medium with $0.5 \mathrm{mg} / \mathrm{L}$ Kinetin and $30 \mathrm{mg} / \mathrm{L}$ Hygromycin) and rooting medium (basic MS medium). The regenerated plants were grown in potting soil under nethouse conditions.

\section{Genotyping of transgenic BT7 rice}

Total DNA of transgenic plants was extracted according to Doyle \& Doyle (1990), using $2 \%$ CTAB solution.

The presence of transgenic expression construct in transgenic BT7 rice plants was confirmed by PCR (Sambrook \& Russel, 2001), with primers Actin-Fw/Actin-Rv, HPTFw/HPT-Rv, Cas9-Fw/Cas9-t-Rv and U6Fw/gRNA-SW-Rv. The PCR was performed with 35 cycles of $94{ }^{\circ} \mathrm{C}, 30 \mathrm{~s} ; 56{ }^{\circ} \mathrm{C}, 30 \mathrm{~s}$ and $72^{\circ} \mathrm{C}, 30 \mathrm{~s}$.

The number of transgene copies was identified by qPCR according to Zhang et al.
(2003). The qPCR performed with 40 cycles of $94{ }^{\circ} \mathrm{C}, 15 \mathrm{~s} ; 60{ }^{\circ} \mathrm{C}, 30 \mathrm{~s}$ and $72{ }^{\circ} \mathrm{C}, 30 \mathrm{~s}$ using HPT-Fw/HPT-Rv primers.

SW14-BT7 mutation was detected by T7 Endonuclease I (T7EI) (Zischewski et al., 2017). A 711-bp fragment of the SW14-BT7 promoter was amplified by PCR (Sambrook and Russel, 2001), using the SW14-tFw/SW14-t-Rv primer pair. PCR products from transgenic and non-transgenic rice plants were mixed at a ratio of $1: 1$ to create heterozygous double strands for detection of mutation (Zischewski et al., 2017). The T7EI enzyme has activity of cutting heterozygous double strands at the mismatch point, which forms two or more DNA fragments. The mixture was processed with T7EI at $37{ }^{\circ} \mathrm{C}$ for 30 minutes. The reaction product was checked by electrophoresis on $2 \%$ agarose gel. PCR products which were cut into 2 DNA bands (410 bp and $301 \mathrm{bp}$ in lengths) were determined as carrying the mutation.

\section{RESULTS}

\section{Designing the expression contruct of the OSSWEET14 promoter-editing complex}

The CRISPR/Cas9 gene editing system works based on the site-specific doublestranded DNA cutting activity of the Cas9 protein (endonuclease) guided by the single guide RNA (sgRNA) molecule. The specificity of the CRISPR/Cas9 complex depends on the 20-nucleotide sequence (crRNA) on the sgRNA molecule (Bortesi \& Fischer, 2015). The designing sgRNA sequence determines the efficiency of the CRISPR/Cas9 complex. In order to demonstrate the role of the three EBE AvrXa7, PthXo3 and Tal5 on the SW14 promoter in the virulence of $\mathrm{Xoo}$ in BT7 rice, the crRNA sequence was designed to induce mutation overlapping the sites of these EBEs.

Using CRISPR-P v2.0 and CCTOP software, 3 crRNA sequences were identified for targeting the position of 3 EBEs, which 
probably form sgRNA molecular with stable secondary constructs (Table 2); of which, crRNA-3 is predicted to be the most effective (total on-score and off-score is 106.3) (Fig. 2).
The crRNA-3 was designed with an additional sticky 3'-end (Table 1) and synthesize artificially (called $g R N A-S W 14$ ) for designing SW14-BT7 - editing T-DNA construct.

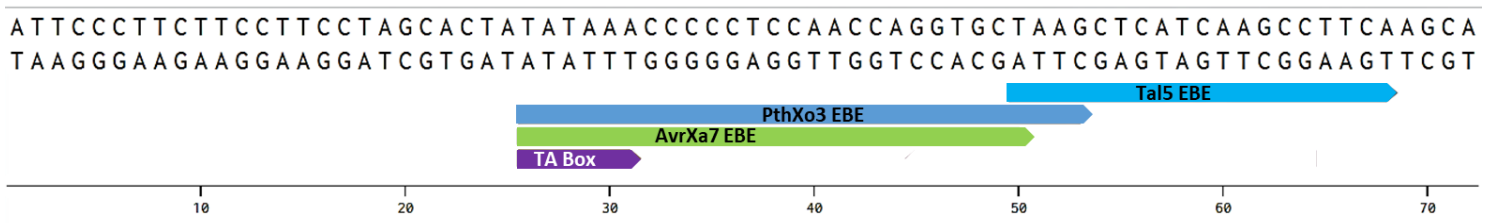

AAGCAAACTCAAGTAGTAGCTGATTACCA TTCGTTTGAGTTCATCATCGACTAATGGT $\frac{100}{1}$

Figure 2. Position of crRNA sequence editing the three EBEs AvrXa7, PthXo3 and Tal5 on promoter SW14-BT7

Table 2. The crRNA for editing SW14-BT7 promoter

\begin{tabular}{|c|l|c|c|c|c|c|c|c|c|}
\hline Name & Sequence (5'-3') & $\%$ GC & TSL & GSL & CBP & TBP & IBP & On-Score & Off-score \\
\hline crRNA1 & $\begin{array}{l}\text { GCTTAGCACCT } \\
\text { GGTTGGAGG }\end{array}$ & 60 & 3 & 0 & 6 & 10 & 0 & 50,6 & 48,1 \\
\hline crRNA2 & $\begin{array}{l}\text { GAGCTTAGCAC } \\
\text { CTGGTTGGA }\end{array}$ & 55 & 3 & 0 & 6 & 10 & 0 & 50,3 & 49,0 \\
\hline crRNA3 & $\begin{array}{l}\text { GGCTTGATGAG } \\
\text { CTTAGCACC }\end{array}$ & 55 & 3 & 0 & 6 & 11 & 0 & 57,9 & 48,4 \\
\hline
\end{tabular}

Note: TSL: total number of loops per sgRNA; GSL: number of loops per crRNA sequence; CBP: number of base pairs that are continuously paired between a crRNA sequence and another sequence on sgRNA; TBP: total number of base pairs paired on sgRNA; IBP: number of base pairs paired in the crRNA sequence; On-score: gRNA activity score (0-100); Off-score: specific score of sgRNA (0-100).

To design the $s g R N A$ expression construct for targeting SW14-BT7, the gRNA-SW14 DNA fragment (Fig. 1) was ligated into the $p E N T R 4-g R N A$ vector pre-treated with $B s a \mathrm{I}$ (Fig. 3A, lane 2) by T4 DNA ligase. The recombinant vector was examined by PCR with two different primer pairs. The results showed that DNA bands with expected sizes of about $1.4 \mathrm{~kb}$ (Fig. 3B, lane 1) and $0.28 \mathrm{~kb}$ (Fig. 3B, lane 3) were observed in PCR products using specific primers for the vector pENTR4-gRNA (pEN-Fw/pEN-Rv) and the gRNA-SW14 - expression construct (U6Fw/gRNA-SW14-Rv), respectively.

gRNA-SW14 expression construct (U6::gRNA-SW14) was ligased into the binary vector pCas9 carrying Cas9 protein expression

construct ([Ubiquitin:Cas9:Nos]) by Gateway system (Fig. 1). The received vector $\mathrm{pCas} 9 / \mathrm{gRNA}$ SW14 was then examined by PCR with primer pairs specific for protein expression construct [Ubiquitin: Nos] (Ubi-Fw/NOSRv), Cas9 (Cas9-Fw/Cas9-Rv), sgRNA expression construct (U6-Fw/Ter-Rv) and gRNA-SW14 (U6-Fw/gRNA-SW14-Rv). The electrophoresis analysis showed that each amplicon corresponding primers has a single $4.3 \mathrm{~kb}, 4.1 \mathrm{~kb}, 0.45 \mathrm{~kb}$ and $0.28 \mathrm{~kb}$ DNA band (Fig. 3C, lanes 1, 3, 5 and 7) on $1 \%$ agarose gel, which were the expected products from the amplified DNA fragments. These results demonstrated that the obtained plasmid carried the $\operatorname{Cas} 9$ gene 
expression construct (controlled by the Ubiquitin promoter) and the gRNA-SW14 expression construct (controlled by the U6 promoter). The recombinant vector was sequenced (Fig. 4) to ensure the correct insertion of the constructs.

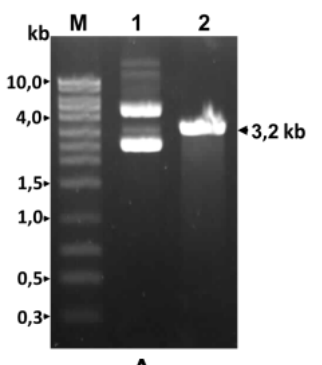

A

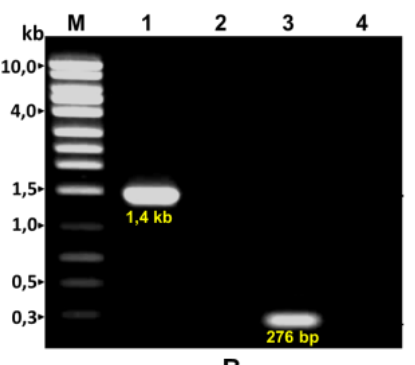

B

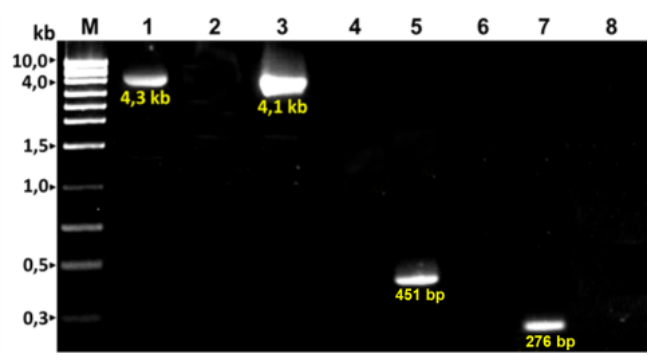

C

Figure 3. Insertion of gRNA-SW14 into the expression construct of T-DNA

Note: (A) Restriction of pENTR4/gRNA by BsaI; lane 1: un-cut vector, lane 2: cut vector. (B) Testing pENTR4/gRNA-SW14 by PCR; lanes 1 and 3: recombinant vector; lanes 2 and 4: negative control (without DNA template); lanes 1 and 2: PCR with pEN-Fw/pEN-Rv primers; lanes 3 and 4: PCR with gRNA-SW14-Fw/gRNA-SW14-Rv primer. (C) Testing vector pCas9/gRNA-SW14 by PCR; lanes 1, 3, 5, 5 and 7: pCas9 / gRNA-SW14; lanes 2, 4, 6 and 8: negative control (without DNA template); lanes 1 and 2: PCR with Ubi-Fw/NOS-Rv primers; lanes 3 and 4: PCR with Cas9-Fw/Cas9-Rv primers; lanes 5 and 6: PCR with U6-Fw/Ter-Rv primers; lanes 7 and 8: PCR with U6-Fw/gRNA-SW14-Rv primers. Lane M: $1 \mathrm{~kb}$ DNA ladder.

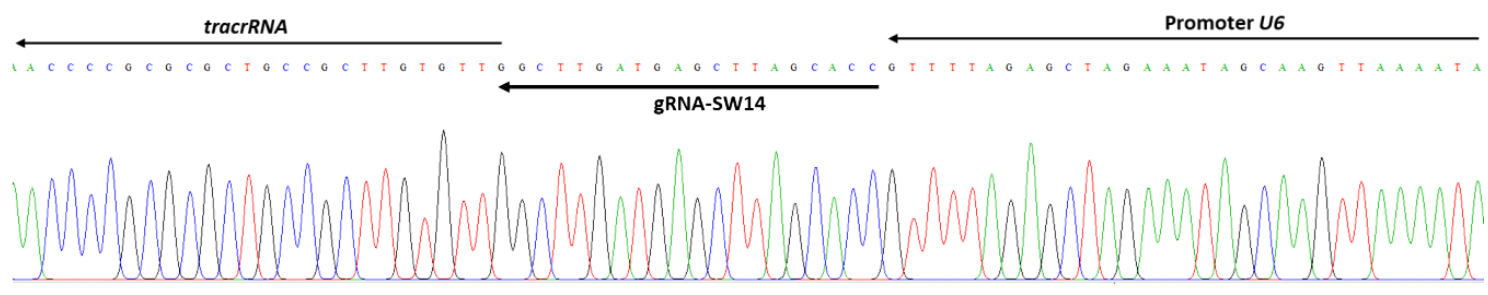

Figure 4. pCas9/gRNA-SW14 sequence.

Note: A part of pCas9/gRNA-SW14 vector was sequenced wiht the Ter-Rv primer. The 20-bp sequence gRNA-SW14 was ligased at the position between U6 promoter and tracrRNA.

In similar studies, the sgRNA expression construct can be engineered in a variety of ways, the most common of which was using overlapping-PCR method. To express the Cas9-sgRNA complex in rice cells, the authors typically design the sgRNA expression construct under the control of the either $U 3$ or $U 6$ promoter and the Cas 9 expression construct under the control of the Ubiquitin promoter, which were located on the same binary vector frame to facilitate the transformation into host cells. This vector system has been shown to be stable, efficient and capable of inducing the inheritable mutations (Ma et al., 2015). In this study, the sgRNA expression construct controlled by the U6 promoter simultaneously targeting 3 EBEs Tal5, AvrXa7 and PthXo3 on the SW14 - BT7 was generated by using $B s a \mathrm{I}$ enzyme and T4 DNA ligase. The construct [U6: gRNA-SW14] and the construct [Ubiquitin: Cas9: NOS] (expressing the $\operatorname{Cas} 9$ protein) were cloned into pCas9 together in order to create a plant transformation vector carrying the construct which expressed the protein-RNA complex modifying promoter $S W 14-B T 7$. 
Transformation of the OsSWEET14 promoter-editing construct into $\mathrm{BT} 7$ rice

To create material for rice transformation experiment, vector pCas9/gRNA-SW14 was transformed into A. tumefaciens EHA105 cells by heat shock method. The transformants were screened by colony PCR with specific primers U6-Fw/Ter-Rv. The electrophoresis results of PCR products from the colonies all gave a single DNA band (Fig. 5, lanes $1-5$ ) with a size of about 0.45 $\mathrm{kb}$ similar to the positive control reaction (Fig. 5, lane 7). This proved that all colonies carried pCas9/gRNA-SW14 construct. A transformant was randomly used for the BT7 rice transformation.

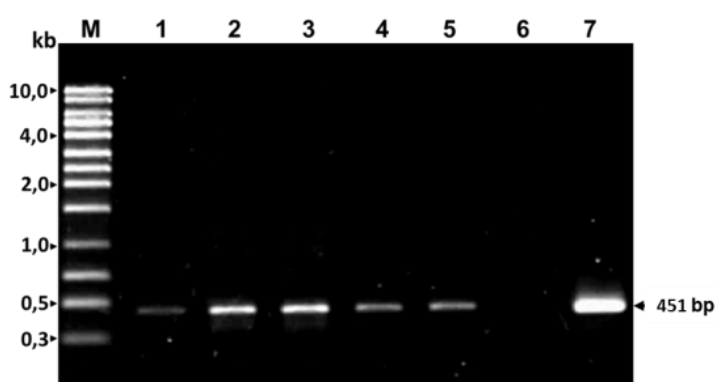

Figure 5. Colony PCR of A. tumefaciens transformants

Note: PCR products with U6-Fw/Ter-Rv primers were electrophoresed on $1 \%$ agarose gel. Lane $\mathrm{M}$ : $1 \mathrm{~kb}$ DNA ladder; lanes 1 - 5: colonies transformed with pCas9/gRNA-SW14; lane 6: negative control (untransformed colonies); lane 7: positive control (pCas9/gRNA-SW14).

A. tumefaciens cells transformed the construct expressing the SW14-BT7-editing CRISPR/Cas9 complex were co-cultured with $\mathrm{BT} 7$ rice calli on in vitro medium supplemented with Acetosyrigone to increase the efficiency of gene transformation. The hygromycin-resistant cell lines growing on selection and shootregeneration media were transferred to root regeneration medium to create complete seedlings (Fig. 6). The results of BT7 rice transformation presented in table 3 showed that the survival rate of calli was quite high, reaching over $75 \%$ in most stages of the gene transfer process. In particular, the regeneration rate of plants after 3 selections reached 54.29\% (38/70 lines regenerated completely). However, only 30 of 38 regenerating plantlets survived affer transferring to potting soil. Finally, from 300 original BT7 rice callus samples, 30 regenerated plants were obtained which grew normally under the nethouse conditions; the achieved regeneration efficiency was $10 \%$. This shoot regeneration rate of $\mathrm{BT7}$ rice callus was similar to that of Pusa Basmati 1 indica rice variety using the same transgenic protocol (Phuong et al., 2015).

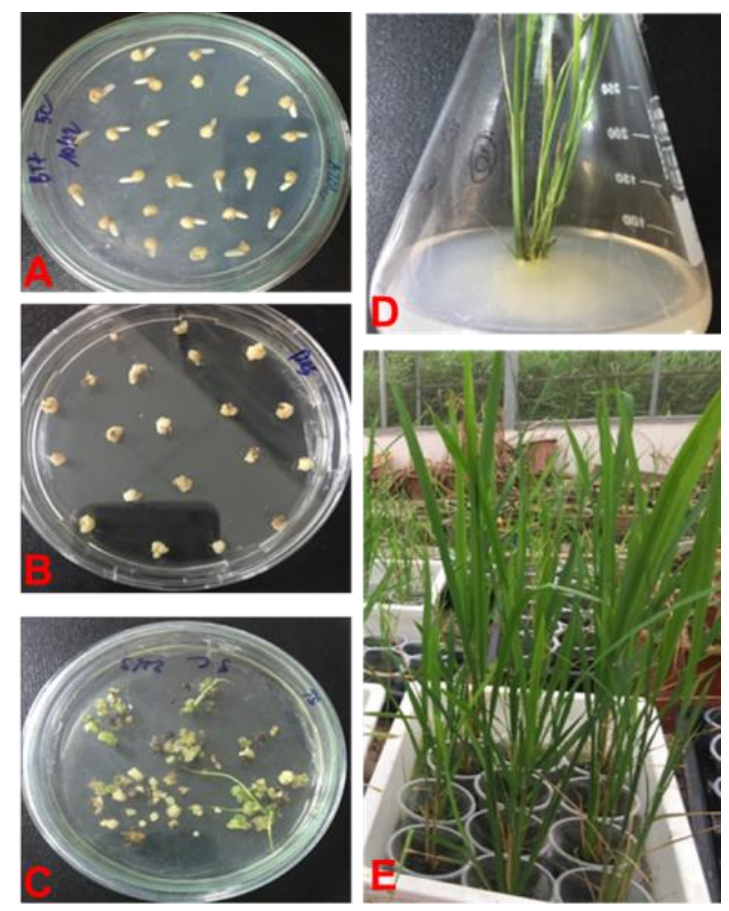

Figure 6. Transformation of SW14-BT7 editing T-DNA construct into BT7 rice variety Notes: (A) Co-culture of calli with A. tumefaciens cells; (B) Selection of calli by $50 \mathrm{mg} / \mathrm{L}$ hygromycin; (C) Shoot regeneration from calli; (D) Root regeneration; (E) Regenerated plants grown in nethouse. 
Table 3. Transformation of pCas9/gRNA-SW14 construct into BT7 rice

\begin{tabular}{|l|c|c|c|c|}
\hline \multicolumn{1}{|c|}{ Stage } & $\begin{array}{c}\text { Total } \\
\text { sample }\end{array}$ & $\begin{array}{c}\text { Number of } \\
\text { surviving/growing samples }\end{array}$ & $\begin{array}{c}\text { Survival/growth } \\
\text { sample rate (\%) }\end{array}$ & Efficiency (\%) \\
\hline Callus induction & 300 & 269 & 89,67 & 89,67 \\
\hline Co-culture & 269 & 250 & 92,94 & 83,33 \\
\hline Selection I & 250 & 200 & 80,00 & 66,67 \\
\hline Selection II & 200 & 150 & 75,00 & 50,00 \\
\hline Selection III & 150 & 80 & 53,33 & 26,67 \\
\hline Pre-regeneration & 80 & 70 & 87,50 & 23,33 \\
\hline $\begin{array}{l}\text { Regeneration, complete } \\
\text { plant production }\end{array}$ & 70 & 38 & 54,29 & 12,67 \\
\hline Nethouse growth & 38 & 30 & 78,95 & 10,00 \\
\hline
\end{tabular}

\section{Genotyping of transgenic BT7 rice plants}

To determine the presence of pCas9/gRNA-SW14 constructs in the genome, total DNA from leaf tissue of 30 lines of regenerated $\mathrm{BT} 7$ rice were extracted and tested by PCR with specific primers (Table 4). The PCR analysis with specific primers for the internal standard gene Actinl showed positive rate of $100 \%$; indicating the good quality of all extracted DNA samples for the PCR experiment. There were 28 of 30 regenerated rice lines showed positive PCR results with specific primers for Hygromycin phosphotransferase (HPT), Cas9 and [U6: gRNA-SW14] construct, reaching the rate of $93.33 \%$ (Table 4). This result suggested that the expression construct of Cas 9 protein and $s g R N A$ (carrying $g R N A-S W 14$ ) were successfully integrated into $\mathrm{BT} 7$ rice genome; the gene transformation efficiency of the whole process reached $9,33 \%$.

In order to ensure the stable function of TDNA constructs, transgenic plants with more than one copy of the transgene are often removed in many plant transformation experiments. Therefore, in this study, regenerated rice plants showing positive PCR results with all four specific primer pairs were further analyzed by qPCR to determine the copy number of the T-DNA construct (through the copy number of HPT gene) in the genome. The analysis indicated that 14/28 regenerated plants had values of $2^{\Delta \mathrm{Ct}}$ from 0.4 to 0.67 , corresponding to single copy of transgene in the genome (Table 4).

Table 4. Genotyping analysis of regenerated BT7 rice lines

\begin{tabular}{|l|c|c|}
\hline \multicolumn{1}{|c|}{ Experiment } & $\begin{array}{c}\text { Number of } \\
\text { tested plants }\end{array}$ & $\begin{array}{c}\text { Number of } \\
\text { positive plants }\end{array}$ \\
\hline PCR with Actin primers & 30 & 30 \\
\hline PCR with HPT primers & 30 & 28 \\
\hline PCR with Cas 9 primers & 28 & 28 \\
\hline PCR with gRNA-SW14 primers & 28 & 28 \\
\hline qPCR identifying plants with one copy of T-DNA & 28 & 14 \\
\hline T7EI assay & 14 & 12 \\
\hline
\end{tabular}

BT7 rice plants with single copy of transgene were used for evaluating the targeted mutation efficiency of the CRISPR/Cas9 complex using T7E1 (T7 endonuclease I assay). The 711-bp fragment containing the recognition site of $g R N A-S W 14$ amplified from SW14-BT7 promoter of each transgenic and non-transgenic rice plant was amplified with SW14-t-Fw/SW14-t-Rv primers (Fig. 7A) and mixed together for T7EI assay (Fig. 7B). Agarose gel electrophoresis of the restriction products 
showed $0.4 \mathrm{~kb}$ and $0.3 \mathrm{~kb}$ DNA bands in 12 of 14 tested transgenic plants (table 4). This result indicated that transformation of pCas9/gRNA-SW14 construct into BT7 rice induced the mutations in the targeted SW14$B T 7$ promoter in transgenic plants, therefore suggested that the SW14-BT7 promoter- editing CRISPR/Cas9-gRNA complex was successfully expressed in BT7 rice cells. Gene editing efficiencies were $85.7 \%$ or $4 \%$ based on the number of transgenic plant harboring one copy of T-DNA (14 lines) or the total number of BT7 seed for callus induction (300 seeds), respectively.
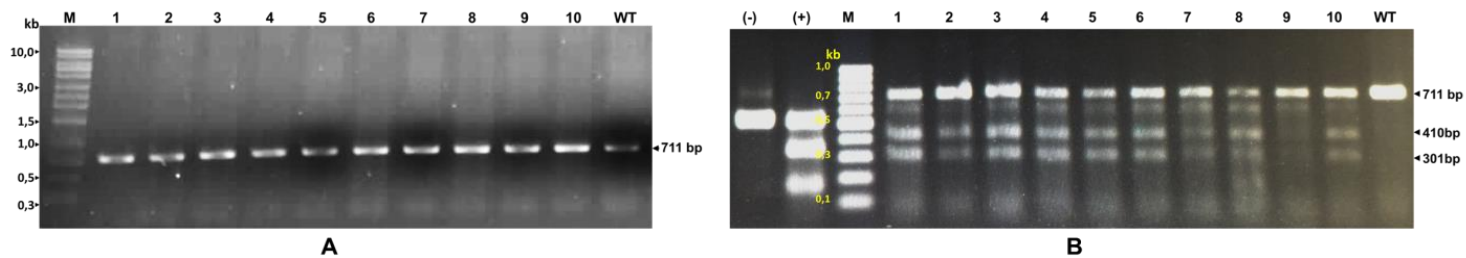

Figure 7. Identification of SW14-BT7 mutation by T7EI.

Note: (A) Amplification of SW14-BT7 fragment with SW14-t-Fw/SW14-t-Rv primers. (B) Mixture of amplicons from transgenic plant and wild-type plant was treated with T7E1. Lanes 1-10: transgenic BT7; lane WT: wild-type BT7; lane (-): negative control; lane (+): positive control; lane M: $1 \mathrm{~kb}(\mathrm{~A})$ and 100 bp (B) DNA ladder.

In general, the success rate of gene transformation in indica rice varieties are much lower than that of japonica rice (SlametLoedin et al., 2014). Although several improved protocols have been published, the efficiency of genetic modification has not been significantly improved. In this study, for the first time, a CRISPR/Cas9-expressing TDNA construct was successfully transformed into a Vietnamese popular (indica) rice variety (BT7 rice) through A. tumefaciens, using calli from mature embryos. In addition, the study has also demonstrated that CRISPR/Cas9 construct induced mutation at targeted site on SW14-BT7 promoter of the transgenic BT7 lines carrying single copy of transgene. Our results showed that it is possible to apply gene editing systems for modifying susceptible genes involved in the virulence of Xoo to host rice plants, and, on that basis, generating a broad-spectrum BLB-resistant BT7 rice in particular and improving the important agronomic traits of Vietnamese major rice varieties in general.

\section{CONCLUSION}

In this study, we have successfully designed protein-RNA complex expression construct for modifying the OsSWEET14 promoter of the Vietnamese elite BT7 rice variety. The gene-editing construct was successfully transformed into BT7 rice through A. tumefaciens with an efficiency of 9,3\%. The regenerated BT7 rice lines have been indentified to carry T-DNA construct in the genome and targeted mutation on the OsSWEET14 promoter through PCR and T7EI analysis. These results are the premise for further studies on the function of OsSWEET14 in the interaction between Xoo and BT7 rice as host plant, thereby leading to the generation of improved rice varieties with high yields and quality and resistance to BLB disease.

Acknowledgements: This study was funded by Ministry of Agriculture and Rural Development under the project "Application of gene editing technology for improving fragrant and BLB resistant traist in the Vienammese major rice varieties" (2017-2020) belong to the Program of Agricultural - Fishery Biotechnology.

\section{REFERENCES}

Blanvillain-Baufumé S., Reschke M., Solé M., Auguy F., Doucoure H., Szurek B., Meynard D., Portefaix M., Cunnac S., Guiderdoni E., Boch J., 2017. Targeted 
promoter editing for rice resistance to Xanthomoanas oryzae pv. oryzae reveals differential activities for SWEET14inducing TAL effectors. Plant Biotechnology Journal, 15(3): 306-317.

Borkar S., Yumlembam R., 2016. Bacterial disease of crop plants. Florida: CRC Press.

Bortesi L., Fischer R., 2015. The CRISPR/Cas9 system for plant genome editing and beyond. Biotechnology Advances, 1(33): 41-52.

Doyle J., Doyle L., 1990. Isolation of plant DNA from fresh tissue. Focus, 12: 13-15.

Hiei Y., Ohta S., Komari T., Kumashiro T., 1994. Efficient transformation of rice (Oryza sativa L.) mediated by Agrobacterium and sequence analysis of the boundaries of the T-DNA. The Plant Journal, 6: 271-282.

Li T., Liu B., Spalding M.H., Weeks D.P., Yang B., 2012. High-efficiency TALENbased gene editing produces diseaseresistance rice. Nature Biotechnology, 30(5): 390-392.

Ma X., Zhang Q., Zhu Q., Liu W., Chen Y., Qiu R., Wang B., Yang Z., Li H., Lin Y., Xie Y., Shen R., Chen S., Wang Z., Chen Y., Guo J., Chen L., Zhao Z., Dong Z., Liu YG., 2015. A robust CRISPR/Cas9 system for convenient, high-effeciency multiplex genome editing in monocot and dicot plants. Molecular Plant, 8(8): 1274-1284.

Phuong N.D., Nghia P.T., Hoi P.X., 2015. Study on genetic transformation of Indica rice with drought-responsive OSNLI-IF gene, Journal of Biotechnology, 13(3): 1-10. (in Vietnamese with English summary).

Sahoo K.K., Tripathi A.K., Pareek A., Sopory S.K., Singla-Pareek S.L., 2011. An improved protocol for efficient transformation and regeneration of diverse indica rice cultivars. Plant Methods, 7(1): 49.
Sam V.H., Ha N.T., Quyen C.L., Phuong N.D., Hoi P.X., 2019. Funtional characterization of the OsSWEET14 gene involved in the infection of blight leaf bacteria in Bacthom 7 variety rice. Science and Technology Jourrnal of Agriculture and Rural Development, 353(2): 13-19. (In Vietnamese with English summary).

Sambrook J., Russel D.W., 2001. A laboratory Manual, 3rd ed. New York: Cold Spring Harbour Laboratory Press, Cold Spring Harbour.

Slamet-Loedin I.H., Chadha-Mohanty P., Torrizo L., 2014. Agrobacteriummediated transformation: rice transformation. Methods in Molecular Biology, 1099: 261-71.

Streubel J., Pesce C., Hutin M., Koebnik R., Boch J., Szurek B., 2013. Five phylogenetically close rice SWEET genes confer TAL effector-mediated susceptibility to Xanthomonas oryzae pv.oryzae. New Phytologist, 200(3): 808-819.

Wang K., 2006. Agrobacterium Protocols. Methods in Mol. Biol 343, 2nd ed. New Jersey: Humana Press, Totowa.

Wang K., 2007. Agrobacterium Protocols (Second edition ed.). Humana Press, New Jersey: Methods in Molecular Biology.

Yang L., Ding J., Zhang C., Jia J, Weng H., Liu W., Zhang D., 2005. Estimating the copy number of transgens in transformed rice by real-time quantitative PCR. Plant Cell Report, 23(10-11): 759-63.

Zhang Y., Zhang D., Li W., Chen J., Peng Y, Cao W., 2003. A novel real-time quatiative PCR method using attached univeral template probe. Nucleic Acids Resource, 31(20): e123.

Zischewski J., Fischer R., Bortesi L., 2017. Detection of on-target and off-target mutations generated by CRISPR/Cas 9 and other sequence-specific nucleases. Biotechnology Advances, 35(1): 95-104. 